\title{
Relationship among chest expansion and scapular index in smartphone users
}

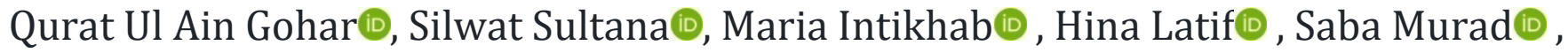 \\ Muhammad Furqaan ${ }^{\circ}$
}

${ }^{1}$ Foundation University Institute of Rehabilitation Sciences, Islamabad, Pakistan.

\begin{abstract}
The objective of this study was to determine whether an association exists between chest expansion and scapular index among smartphone users who use smartphones greater than four hours. The study was a cross sectional correlational analytical study conducted in Rawalpindi / Islamabad community after approval from the ethical review committee. The duration of study was from June 2019 to February 2020. The total participants were 245 (64 males and 181 females), aged 18 to 40 years who spend their time on smartphones greater than 4 hours. The tools used for data collection procedure were Digital Vernier caliper, measuring tape and self-structured questionnaire. Vernier caliper was used to measure rounded shoulders by calculating Scapular index and chest expansion was calculated using measuring tape. Significant relation was found between chest expansion and right scapular index with screen time among smartphone users with $\mathrm{p}$ value $<0.05$. Statistical analysis of this study highlights that there is a significant relationship between duration of smartphone usage and scapular index with $\mathrm{p}$ value of right scapula is 0.036 and left scapula are 0.037 but non-significant relationship exists between chest expansion and smart phone usage duration. Our study supports that the position of shoulder during smartphone usage and duration of smartphone more than 4 hours affects scapular index and have a negative impact on their posture which cause rounded shoulders as compared to those who spend less time.
\end{abstract}

Keywords. Chest expansion, duration, scapular index, smart phone.

\section{Introduction}

Smartphones are modern devices used globally for communication and have diverse software applications, major operating systems with powerful computing capacity. Smartphone usage among people has risen dramatically in the 21st century (Lee et al., 2015). Frequent use of a smartphone should be no more than 4 hours with good positioning and posture and also correctly handling devices is also important for the respiratory system. With increased use of such smartphone devices structural problems, as well as musculoskeletal pain patterns like neck/shoulder pain and breathing dysfunction also increases. Scapular index is also reduced while using a smartphone, leading to poorer forward head posture and rounded shoulders compared to those who use smartphones for less time per day (Jung et al., 2016)
Prolong use of smartphones is dominant among young adults due to advancement in smartphone applications which contribute to its addiction which in turn increases the ratio of musculoskeletal problems (Eitivipart et al., 2018). The posture we adapt during smartphone usage is holding the device with one or two hands below the eye level and using the thumb to touch the screen. This uncomfortable posture of upper extremity maintained by us for prolonged time, contributes as a cardinal factor in musculoskeletal problems (Salvi \& Battin, 2018). Scapular index which indicates the relationship of breath to the length is used to assess the shape of the scapula (Abdelhameed \& Abdel-Aziem, 2016). Scapula forms the basis of upper extremity posture, and any alteration in the position of the scapula is a predisposing factor for musculoskeletal aches and pain. Unnecessary usage of hand devices cause different faulty posture such as forward neck posture,

$凶$ Q. U. A. Gohar, e-mail: quratulaingohar509@gmail.com

Received: November 03, 2021 - Accepted: December 17, 2021 - Published: December 31, 2021

To Cite: Gohar, Q.U.A., Sultana, S., Intikhab, M. Latif, H., Murad, S., Furqaan, M. (2021). Relationship among chest expansion and scapular index in smartphone users. TurkJ Kinesiol, 7(4), 105-111. DOI: 10.31459/turkjkin.1014693 
slouched posture or rounded shoulders (Vora et al., 2019). Regarding the relationship between faulty posture and shoulder disorder, some investigations have demonstrated influence of thoracic posture on the functions of the scapula and shoulder (Kim \& Kim, 2016). The alterations in scapular positioning can have an effect on shoulder function (Koseki et al., 2019). Furthermore, scapular positioning is hypothesized to be a direct relationship to scapular stability and the generation of peripheral and respiratory muscular forces. As the addiction of phone usage increases it causes imperfect posture and shoulder disorder, the Cranio-Vertebral angle decreases, which is a sign of a forward head posture (Zafar et al., 2018). The extensive use of smart phones among children may lead them to become addicted, which is considered one of the greatest global health concerns among people (Balakrishnan et al., 2016). Revolutionize pose and respiratory dysfunction is depending on the position and time spent on Smartphone usage that effecting the breathing mechanism and reducing chest movements. Cervicothoracic mobility and change in posture especially poor sitting interfere with breathing mechanics. Altered diaphragm function leads to core muscle instability which will further progress to musculoskeletal disorders. Chest expansion is the measurement of thoracic girth after maximal inspiration and at the end of maximal expiration and these measurements are used for treatment effectiveness, and progression of disease with regards to chest wall mobility and respiratory muscle function and indicator of many musculoskeletal problems. The normal chest expansion shouldn't be less than $3 \mathrm{~cm}$, the reduction in chest expansion leads to low lung function and impaired breathing mechanism. The increase in prolong use of Smartphones may cause reduction in chest movements which further causes complexity in breathing mechanism (Kim et al., 2013). Prolong use of phones greater than four hours has raised concerns about upper and lower back posture. Duration of Smartphone should be carefully monitored in young population in order to avoid further future problems related to posture as well as pulmonary function (Janssens et al., 1999). However, as per available literature, the data in Pakistani population is lacking, therefore this study aims to determine whether an association exists between chest expansion and Scapular index among smartphone users in population of twin cities of Pakistan.

\section{Methods}

The design selected for this was a cross sectional correlational analytical study conducted in Rawalpindi/Islamabad community. The total sample was 245 (64 males and 181 females) aged 18 to 40 years, who were recruited for this study. The participants were screened for inclusion and exclusion criteria, smokers and any type of postural abnormalities were excluded if any positive sign or symptom was found. The ethical approval for this study was obtained from the Ethical Review Committee of the Foundation University Medical College, Islamabad (No: FF/FUMC/215 phy/19; Date: October 15, 2019). All the subjects were informed about the purpose and nature of the study and informed consent was obtained before participation in the research process. The tools used for data collection procedure included the self-structured questionnaire along with digital Vernier caliper and measuring tape. The Digital Vernier caliper was utilized to measure rounded shoulders by calculating Scapular index. The intra and inter class reliability is 0.64-0.86 and 0.7-0.9.the normal value range is $65.80-90.00 \mathrm{~mm}$.

Rounded shoulders were assessed by measuring scapular index. Participants were made to stand in a relaxed posture with hands hanging by their sides. Coracoid process and sternal notch were palpated and marked anteriorly, whereas the posterior boundary of acromion process and adjacent thoracic vertebral spine were palpated and marked. Vernier caliper was used to measure the distance between the above-mentioned points anteriorly and the distance between the points posteriorly.

The formula used for calculating scapular index was:

SI= Distance between acromion process and sternal notch /_Distance between acromion process and spine

Chest expansion was measured with measuring tape: intra class correlation coefficient ranged from 0.81 to 0.9 . The normal value was $(4.0$ to $7.0 \mathrm{~cm})$.

\section{Statistical Analysis}

Data analysis was done by using SPSS Version 20 . The total sample size was 245 (64 males and 181 females). Descriptive statistics were recorded in terms of mean and standard (Gender and age). Based upon results of Kolmogorov-Smirnov test for chest expansion and scapular index (right and left), the assumption of normality was violated. It suggested the use of non-parametric (Mann Whitney U) test to 
find the correlation. Spearman correlation was utilized to determine correlation between study variables that are scapular index Right/left and chest expansion.

\section{Results}

Our study determines weak positive relation between scapular index and chest expansion with $r$ value for right scapula and chest expansion is 0.181 (Table 1). Out of total sample $23.7 \%$ student use smart phone continuously greater than 4 hours, $71.8 \%$ use intermittent greater than 4 hours while the last group include $4.5 \%$ students who use smartphone less than 4 hours (Figure 1). The consequences show significant relation between Right and Left scapular index (Table 2) and also show significant relation between chest expansion and right scapular index with screen time among Smartphone users greater than 4 hours (Figure 2).

Statistical analysis of this study highlights that there is a significant relationship between duration of smartphone usage and scapular index with p value of right scapula is 0.036 and left scapula are 0.037 but non-significant relationships between chest expansion and phone usage duration (Table 3). The post hoc comparison of scapular index shows significant relation between $>4$ hours, intermittent smartphone users and $<4$ hours smartphone users with $p$-value for right scapula is 0.021 and left scapula $p$-value 0.014 respectively (Table 4 ).

Table 1

Correlation of Chest Expansion and Scapular Index.

\begin{tabular}{lcc}
\hline Variables & $r$ & $p$ \\
\hline Chest Expansion - Scapular Index (Rt.) & 0.181 & $0.004^{*}$ \\
Chest Expansion - Scapular Index (Lt.) & 0.125 & 0.052 \\
\hline
\end{tabular}

Table 1 showing the correlation of chest expansion and scapular index, with right scapula $\mathrm{r}$ value was 0.181 while $p$-value is 0.004 and with left scapula $r$ value was 0.125 while $p$-value was 0.052 .

Table 2 showing significant results for analysis of screen time duration with right and left scapular index with $P$ value 0.036 and 0.037 respectively.

Table 3 showing analysis of screen time duration with chest expansion and scapular index, $p$-value for chest expansion is 0.633 with median and IQR $3.5 \pm$ 2.3, $3.0 \pm 02$ and $3.0 \pm 1.3$ respectively. The $p$-value for scapular index, value for right scapula is 0.036 with median and IQR 63.11 $\pm 11.17,62.39 \pm 14.64$ and $55.77 \pm 7.34$ respectively. Value for left scapula is 0.037 with median and IQR $64.70 \pm 13.18,63.00 \pm$ 13.39 and $58.69 \pm 9.86$ respectively.

\section{Table 2}

Analysis of duration of screen time with scapular index (Right) and Scapular index (Left).

\begin{tabular}{lcccc}
\hline Variables & $\begin{array}{c}\text { Greater than 4 Hours } \\
\text { continuously [Median (IQR)] }\end{array}$ & $\begin{array}{c}\text { Greater than 4 Hours } \\
\text { Intermittently [Median (IQR)] }\end{array}$ & $\begin{array}{c}\text { Less than 4 Hours } \\
\text { [Median (IQR)] }\end{array}$ & $p$ \\
\hline Scapular Index (Rt) & $63.11 \pm 11.17$ & $62.39 \pm 14.64$ & $55.77 \pm 7.34$ & $0.036^{*}$ \\
Scapular Index (Lt) & $64.70 \pm 13.18$ & $63.00 \pm 13.39$ & $58.69 \pm 9.86$ & $0.037^{*}$ \\
\hline$* p<.05 ;$ Mean \pm SD & & &
\end{tabular}

\section{Table 3}

Analysis of duration of screen time with chest expansion, scapular index (Rt.) and Scapular index (Lt.).

\begin{tabular}{|c|c|c|c|c|}
\hline Variables & $\begin{array}{c}\text { Greater than } 4 \text { Hours } \\
\text { continuously [Median (IQR)] }\end{array}$ & $\begin{array}{c}\text { Greater than } 4 \text { Hours } \\
\text { Intermittently [Median (IQR)] }\end{array}$ & $\begin{array}{l}\text { Less than } 4 \text { Hours } \\
\text { [Median (IQR)] }\end{array}$ & $p$ \\
\hline Chest Expansion & $3.5 \pm 2.3$ & $3.0 \pm 02$ & $3.0 \pm 1.3$ & 0.633 \\
\hline Scapular Index (Rt) & $63.11 \pm 11.17$ & $62.39 \pm 14.64$ & $55.77 \pm 7.34$ & $0.036^{*}$ \\
\hline Scapular Index (Lt) & $64.70 \pm 13.18$ & $63.00 \pm 13.39$ & $58.69 \pm 9.86$ & $0.037^{*}$ \\
\hline
\end{tabular}


Table 4

Post hoc Comparison of scapular index between greater than 4 hours intermittent Smartphone users \& less than 4 hours' Smartphone users

\begin{tabular}{lccc}
\hline Variables & $\begin{array}{c}\text { Greater than } 4 \text { Hours Intermittently } \\
{[\text { Median (IQR)] }}\end{array}$ & $\begin{array}{c}\text { Less than 4 Hours } \\
{[\text { Median (IQR)] }}\end{array}$ & $p$ \\
\hline Scapular Index (Rt) & $62.39 \pm 14.64$ & $55.77 \pm 7.34$ & $0.021^{*}$ \\
Scapular Index (Lt) & $63.00 \pm 13.39$ & $58.69 \pm 9.86$ & $0.014 *$ \\
$* \mathrm{p}<.05 ;$ Mean \pm SD & & &
\end{tabular}

Table 4 shows significant results for post hoc comparison of scapular index between $>4$ hours, intermittent Smartphone users and $<4$ hours Smartphone users with $p$-value for right scapula is 0.021 and left scapula $p$-value 0.014 respectively.

Figure 1 presents smartphone usage distribution showing Screen time into three classes in which $23.7 \%$ student use smart phone continuously greater than 4 hours, $71.8 \%$ use intermittent greater than 4 hours while the last group include $4.5 \%$ students who use Smartphone less than 4 hours.

Figure 2 shows analysis of screen time duration with chest expansion and scapular index, $\mathrm{P}$ value for chest expansion is 0.633 with median and IQR $3.5 \pm$ $2.3,3.0 \pm 02$ and $3.0 \pm 1.3$ respectively. $P$ value for scapular index, value for right scapula is 0.036 with median and IQR 63.11 $\pm 11.17,62.39 \pm 14.64$ and $55.77 \pm 7.34$ respectively. Value for left scapula is 0.037 with median and IQR $64.70 \pm 13.18,63.00 \pm$ 13.39 and $58.69 \pm 9.86$ respectively.

The consequences shows that prolong use of Smartphone greater than 4 hours arises musculoskeletal problems and has negative impact on our posture as compared to those who spend less time. It was well-known that subjects who showed a high level of mobile phone addiction had a decreased Scapular Index; there is reciprocity of rounded shoulders and mobile phone addiction in the participants. It divulged that smart phone prolong usage affect scapular index and posture thus contribute in various musculoskeletal as well as respiratory problems.

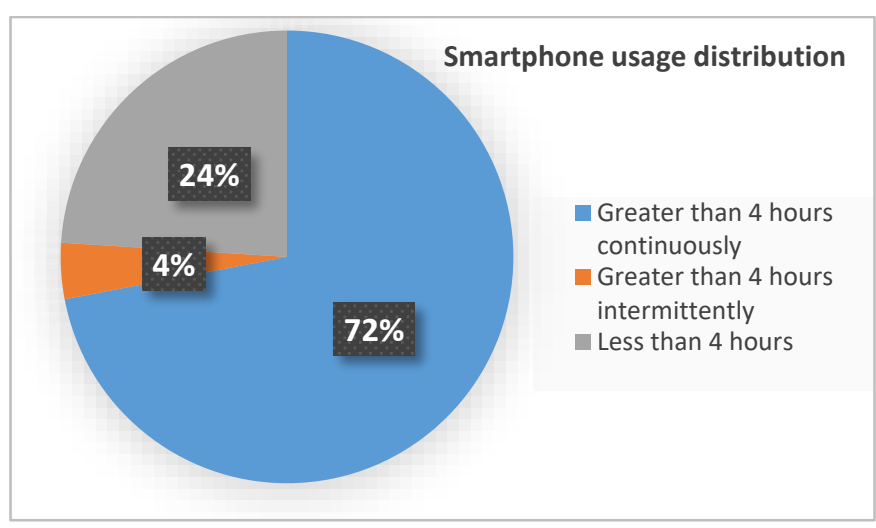

Figure 1. Smartphone usage distribution.

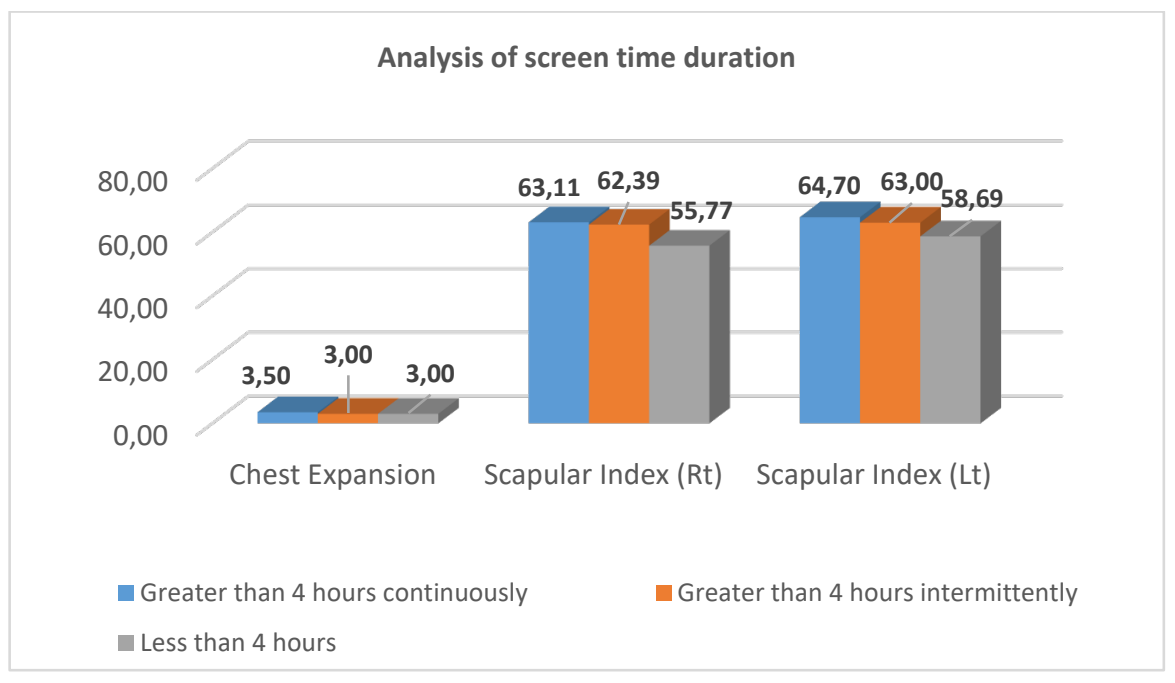

Figure 2. Analysis of screen time duration. 


\section{Discussion}

The long-term use of smartphone had negative impact on posture, mobility impairments, decreases the ideal function of the body as well as respiratory function. Many relatable studies were conducted on chest expansion and scapular index among smart phone users but no one debated about their mutual dependency and relationship. Current study investigated the relationship between Scapular Index Right \Left and chest expansion among Smartphone users. The result revealed weak positive and significant correlation between chest expansion and right scapular index with $r$ value for right scapula and chest expansion is 0.181 and left scapula and chest expansion with $p$ values of 0.004 and 0.052 respectively. A Korean study which was conducted in 2015 supported the conclusion of this study, which indicated that usage of smartphones for extended periods of time may cause bad impact on posture as well as respiratory function (Jung et al., 2016). Contrary to this, another study was conducted in 2016 indicated negative association between smartphone usage posture and rounded shoulders (Kim \& Kim, 2016).

Another study "Correlation of Mobile Phone Addiction Scale (MPAS) Score With Cranio-vertebral Angle, Scapular Index And Beck's Depression Inventory Score In Young Adults" was conducted in 2018 supported that the subjects who showed a high level of mobile phone addiction had a decreased Scapular Index; there is reciprocity of rounded shoulders and mobile phone addiction in the participants.(Salvi \& Battin, 2018) There is recent article of 2019, published in cogent engineering which supported the results of this study by proving, smart phones negatively affect the postures, contrary to this an observational descriptive study suggested alteration and pain in the scapular region was not related to an individual's mobile phone use and can have various other contributory factors (Vora et al., 2019).

The recognized factors for the smartphone usage may contribute to musculoskeletal disorders. This study post hoc comparison reported non-significant relationship between time duration and scapular index $>4$ hours continuously and > 4hours intermittently with $\mathrm{p}$ value of 0.620 and 0.598 respectively. In contrast, study was conducted in Korea with title of angle of head flexion while spending time on using smart phone, accomplished that text messaging, which is one of the most customarily used application, could be a main causative factor to the incidence of musculoskeletal pain of substantial smart phone users (Kim \& Kim, 2016).

The impact of cell phones and their effects on human health and life are still being verified and studied. This study post hoc analysis between smart phone usage duration and scapular index revealed significant relationship with $\mathrm{p}$ values of right scapula is 0.004 and left scapula is 0.013 respectively. Additional supported study published in an International Journal of Physical Education, Sports and Health 2016 conducted in Malaysia by Tai Fen depicted that on the wide usage of handheld devices and their influence on musculoskeletal disorders in which researchers recognized that extended use of cell phone is known to cause symptoms of musculoskeletal disorder keeping this into consideration, more study should be done in the future to create awareness among cell phone user concerning the seriousness of this problem (Balakrishnan et al., 2016).

Occurrence of dire posture are the lack of instruction about ergonomic positions when using a smartphone. Results of 2017 research persistent with the current study which concluded that frequent use of smart phones will negatively affect posture, as well as respiratory biomechanics. Therefore, education on proper posture while using the smart phone and effects of prolonged usage of smartphone are necessary to preserve normal functions (Alonazi, 2017).

This study post hoc comparison statistics between scapular index and phone usage duration > intermittently 4 hours and $<4$ hours reveals significant relationship with p value of Right scapula is 0.021 and Left scapula is 0.014 while in comparison study conducted in India under title of Effect of Posture Correction Exercises and Ergonomic Advices in People Having Postural Abnormalities among Chronic Smartphone Users in which Intra-group comparison showed that cranio-vertebral angle and scapular index were statistically extremely significant, cranio-vertebral angle $(p=<0.0001)$, scapular index $(\mathrm{p}=<0.0001)$ in chronic smart phone users having postural abnormalities. Therefore, Posture correction exercises and ergonomic advices were significantly effective in correcting postural abnormalities (Shete \& Shah, 2019; Dalawala \& Pimpale, 2017).

Statistical analysis of this study highlighted that there is significant relationship between duration of smart phone usage and scapular index with $p$ value of right scapula is 0.036 and left scapula are 0.037 but non-significant relationships between chest 
expansion and phone usage duration. Recent year another study published in Iranian journal of ergonomics indicated a significant relation between high users and low users of smartphones with position of shoulder, smartphone usage duration has significant effect on scapular index thus supports our study. Ignorance of ideal guidelines, commonly smartphone use which lead to postural problems (Masoumi \& Akoochakian, 2019).

Another study published in International journal Assessment of Co-Morbid Factors Associated with Text-Neck Syndrome among Mobile Phone Users conducted in India reported that neck musculoskeletal disorder is related to thoracic spine kyphosis and rounded shoulder posture (Vijayakumar et al., 2018).

In the literature, patients in a standing position chest expansion was measured, even if other position were also used. In the current study subjects were standing during measurements. Certainly, handling of the tape measure seemed easier in this position. Moreover, another study supported that thoracic breathing is significantly enhanced in this position compared with abdominal breathing and this difference is highlighted when the subjects breathe with larger volumes(Debouche et al., 2016).

A study Scapula kinematics differ by body mass index conducted in Aug 2013 by Miti Gupta highly supported that the biomechanical changes due to increased arm mass in individuals with high body mass index (BMI) may lead to altered scapular motions at the shoulder joint. Data were analyzed with a mixed-model ANOVA with Group and Elevation Angle the between- and within-subject factors, respectively. It resulted that the group higher the BMI had significantly greater scapular upward rotation at $120^{\circ}$ for both the weighted, unweighted and tasks than group with lower BMI. In this study altered scapula-thoracic movement was found in individuals with higher BMI had (Gupta et al., 2013).

This study statistical analysis proved that there is weak positive relation between chest expansion and scapular index of right and left scapula. The results also showed that prolong use of smart phone affect scapular index and contribute in various musculoskeletal problems. So, it negatively affects both posture and respiratory system. These finding can be utilize in community awareness. For health consideration, we need to give attention towards phone usage duration as well as our posture.

The limitations of this study were small sample size. Single tool was utilized to measure both the posture and chest expansion. Future studies with greater sample size and multiple variables should be conducted to establish the correlation significantly and to generalize the results for twin cities.

\section{Conclusion}

This study concluded that prolonged use of smartphones affects scapular index and contributes to various musculoskeletal problems. Our study supports that Smartphone with position of shoulder and those who spend more than 4 hours continuously on their Smartphone have a negative impact on their posture causing rounded shoulders. This study also concluded that duration of smart phone usage do not affect the chest expansion.

\section{Acknowledgement}

Thanks to Allah Almighty who blessed us wisdom, strength, and knowledge to accomplish my research work and for speaking to my spirit when I felt like giving up. We would also like to thank our teachers who supported and guided us at each step of our project. This dissertation would not have been possible without the patience and generosity of our mentors and also our Foundation University Islamabad.

\section{Disclaimer: None}

Conflict of interest: The authors declare no conflicts.

Funding Disclosure: This research work was funded by ourselves.

\section{References}

Abdelhameed, A. A. \& Abdel-Aziem, A. A. (2016). Exercise training and postural correction improve upper extremity symptoms among touchscreen smartphone users. Hong Kong Physiotherapy Journal, 35, 37-44.

Alonazi, A. (2017). The effects of frequent smartphone use on children's upper posture and pulmonary function [Doctoral dissertation, Loma Linda University]. Loma Linda University Electronic Theses, Dissertations \& Projects. https://scholarsrepository.llu.edu/etd

Balakrishnan, R., Chinnavan, E., \& Feii, T. (2016). An extensive usage of hand held devices will lead to musculoskeletal disorder of upper extremity among student in AMU: A survey method. Int J Phys Educ Sports Health, 3(2), 368-372.

Dalawala, P. J. \& Pimpale, S. K. (2017). Assessment of craniovertebral angle and depression in heavy 
smartphone users. Indian Journal of Physiotherapy \& Occupational Therapy, 11(4), 88-92.

Debouche, S., Pitance, L., Robert, A., Liistro, G., \& Reychler, G. (2016). Reliability and reproducibility of chest wall expansion measurement in young healthy adults. Journal of Manipulative and Physiological Therapeutics, 39(6), 443-449.

Eitivipart, A. C., Viriyarojanakul, S., \& Redhead, L. (2018). Musculoskeletal disorder and pain associated with smartphone use: A systematic review of biomechanical evidence. Hong Kong Physiotherapy Journal, 38(02), 7790.

Gupta, M., Dashottar, A. \& Borstad, J. D. (2013). Scapula kinematics differ by body mass index. J Appl Biomech, 29(4), 380-385.

Janssens, J.-P., Pache, J.-C., \& Nicod, L. (1999). Physiological changes in respiratory function associated with ageing. Eur Respir J, 13(1), 197-205.

Jung, S. I., Lee, N. K., Kang, K. W., Kim, K., \& Do, Y. L. (2016) The effect of smartphone usage time on posture and respiratory function. J Phys Ther Sci, 28(1), 186-189.

Kim, E.-K. \& Kim, J. S. (2016). Correlation between rounded shoulder posture, neck disability indices, and degree of forward head posture. J Phys Ther Sci, 28(10), 29292932.

Kim, Y.-G., Kang, M.-H., Kim, J.-W., Jang, J.-H., \& Oh, J.-S. (2013). Influence of the duration of smartphone usage on flexion angles of the cervical and lumbar spine and on reposition error in the cervical spine. Physical Therapy Korea, 20(1), 10-17.

Koseki, T., Kakizaki, F., Hayashi, S., Nishida, N., \& Itoh, M. (2019). Effect of forward head posture on thoracic shape and respiratory function. J Phys Ther Sci, 31(1), 63-68.

Lee, S., Kang, H., \& Shin, G. (2015). Head flexion angle while using a smartphone. Ergonomics, 58(2), 220-226.

Masoumi, A. S. and M. Akoochakian (2019). The effect of duration of smartphone use on head and shoulders posture of young adults aged 20-35 years. Iran J Ergon, $7(2), 62-71$

Salvi, R., \& Battin, S. (2018). Correlation of mobile phone addiction scale (MPAS) score with craniovertebral angle, scapular index and beck's depression Inventory score in young adults. Int J Physiother, 5(1), 7-12.

Shete, M. G., \& Shah, R. (2019). Effect of posture correction exercises and ergonomic advices in people having postural abnormalities among chronic smartphone users. International Journal of Health Sciences \& Research, 9(7), 121-125.

Vijayakumar, M., Mujumdar, S., \& Dehadrai, A. (2018). Assessment of co-morbid factors associated with textneck syndrome among mobile phone users. IJSRST, 4(9), 38-46.

Vora, T., Goda, K., Dhole, N., Verma, C., \& Vichare, S. (2019). Study of the association between scapular position and mobile phone usage in college students: An observational descriptive study. Physiother - J Indian Assoc Physiother, 13(1), 18-22.

Zafar, H., Albarrati, A., Alghadir, A. H., \& Iqbal, Z. A. (2018). Effect of different head-neck postures on the respiratory function in healthy males. BioMed Research International, 2018, 4518269. 